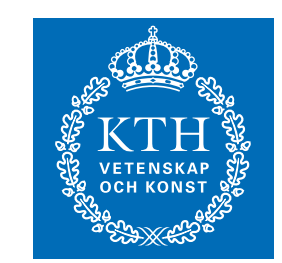

KTH Electrical Engineering

\title{
On the Impact of Spatial Correlation and Precoder Design on the Performance of MIMO Systems with Space-Time Coding
}

\author{
Proceedings of IEEE International Conference on \\ Acoustics, Speech, and Signal Processing (ICASSP)
}

April 19 - 24, Taipei, Taiwan, 2009

(C) 2009 IEEE. Published in the IEEE 2009 International Conference on Acoustics, Speech, and Signal Processing (ICASSP 2009), scheduled for April 19 - 24, 2009 in Taipei, Taiwan. Personal use of this material is permitted. However, permission to reprint/republish this material for advertising or promotional purposes or for creating new collective works for resale or redistribution to servers or lists, or to reuse any copyrighted component of this work in other works, must be obtained from the IEEE. Contact: Manager, Copyrights and Permissions / IEEE Service Center / 445 Hoes Lane / P.O. Box 1331 / Piscataway, NJ 08855-1331, USA.

Telephone: + Intl. 908-562-3966.

\section{EMIL BJÖRNSON, BJÖRN OTTERSTEN, AND EDUA'RD JORSWIECK}

Stockholm 2009

\author{
KTH Royal Institute of Technology \\ ACCESS Linnaeus Center \\ Signal Processing Lab
}

IR-EE-SB 2009:002, Revised version with minor corrections 


\section{ON THE IMPACT OF SPATIAL CORRELATION AND PRECODER DESIGN ON THE PERFORMANCE OF MIMO SYSTEMS WITH SPACE-TIME CODING}

\author{
Emil Björnson, Björn Ottersten \\ ACCESS Linnaeus Center \\ Signal Processing Lab \\ Royal Institute of Technology (KTH) \\ \{emil.bjornson,bjorn.ottersten\}@ee.kth.se
}

\author{
Eduard Jorswieck \\ Chair of Communication Theory \\ Communications Laboratory \\ Dresden University of Technology \\ jorswieck@ifn.et.tu-dresden.de
}

\begin{abstract}
The symbol error performance of spatially correlated multi-antenna systems is analyzed herein. When the transmitter only has statistical channel information, the use of space-time block codes still permits spatial multiplexing and mitigation of fading. The statistical information can be used for precoding to optimize some quality measure. Herein, we analyze the performance in terms of the symbol error rate (SER). It is shown analytically that spatial correlation at the receiver decreases the performance both without precoding and with an SER minimizing precoder. Without precoding, correlation should also be avoided at the transmitter side, but with an SER minimizing precoder the performance is actually improved by increasing spatial correlation at the transmitter. The structure of the optimized precoder is analyzed and the asymptotic properties at high and low SNRs are characterized and illustrated numerically.
\end{abstract}

Index Terms-Linear Precoding, Majorization, MIMO Systems, Orthogonal Space-Time Block Codes, Symbol Error Rate.

\section{INTRODUCTION}

The use of multiple antennas at the transmitter and receiver sides in wireless communication systems has the potential of dramatically increasing the throughput in environments with sufficient scattering. With full channel state information (CSI) available at both the transmitter and receiver sides, low complexity receivers exist that can realize this throughput increase [1]. Unfortunately, full CSI at the transmitter is unrealistic in many fast fading scenarios since it would require a prohibitive feedback load. The long-term channel statistics can, on the other hand, usually be considered as known since they vary much slower than the channel realization. When required, instantaneous CSI can also be achieved at the receiver from training signalling [2].

The primary use of multiple antennas is to increase the reliability by mitigating fading and to increase throughput by transmitting several data streams in parallel, cf. [3]. When the transmitter is completely unaware of the channel, one common way of achieving these two goals is to use orthogonal space-time block codes (OSTBCs) [4]. Herein, we assume that the transmitter has statistical CSI, which makes it possible to adapt the coding to the channel statistics. Linear precoding of OSTBCs was proposed in [5], and has recently been analyzed in [6] and [7] with respect to the symbol error rate (SER).

This work is supported in part by the FP6 project Cooperative and Opportunistic Communications in Wireless Networks (COOPCOM), Project Number: FP6-033533. Björn Ottersten is also with securityandtrust.lu, University of Luxembourg.
In this paper, we consider the SER performance of spatially correlated multiple-input multiple-output (MIMO) systems with OSTBCs. The influence of precoding and correlation on the SER will be analyzed in terms of Schur-convexity [8]. Previously, the Chernoff bound on the SER has been analyzed in this manner assuming a certain type of modulation [9]. The error performance was shown to decrease with increasing correlation in most scenarios, except when an SER minimizing precoder was employed; then, the performance improves as the correlation increases at the transmitter side. Herein, the results of [9] are generalized to cover a much larger class of modulation schemes and to consider the exact value of the SER.

First, an introduction to the problem and to the mathematical technique of majorization will be given. Then, we will review the SER expressions for PAM, PSK, and QAM constellations, and show how they all share the same general structure. This result will be exploited in the analysis of the SER, where we consider the case of space-time coded transmissions without precoding and with SER minimizing precoding. The impact of spatial correlation on the SER will be derived analytically and then illustrated numerically.

\subsection{Notation}

Vectors and matrices are denoted with boldface in lower and upper case, respectively. The Kronecker product of two matrices $\mathbf{X}$ and $\mathbf{Y}$ is denoted $\mathbf{X} \otimes \mathbf{Y}$. The vector space of dimension $n$ with nonnegative and real-valued elements is denoted $\mathbb{R}_{+}^{n}$. The Frobenius norm of a matrix $\mathbf{X}$ is denoted $\|\mathbf{X}\|$.

\section{SYSTEM MODEL AND PRELIMINARIES}

We consider a correlated Rayleigh flat-fading channel with $n_{T}$ transmit antennas and $n_{R}$ receive antennas. The channel is represented by the matrix $\mathbf{H} \in \mathbb{C}^{n_{R} \times n_{T}}$ and is assumed to follow the Kronecker model

$$
\mathbf{H}=\mathbf{R}_{R}^{1 / 2} \widetilde{\mathbf{H}} \mathbf{R}_{T}^{1 / 2}
$$

where $\mathbf{R}_{R} \in \mathbb{C}^{n_{R} \times n_{R}}$ and $\mathbf{R}_{T} \in \mathbb{C}^{n_{T} \times n_{T}}$ are the positive semidefinite correlation matrices at the receiver and transmitter side, respectively. $\widetilde{\mathbf{H}} \in \mathbb{C}^{n_{R} \times n_{T}}$ has i.i.d. complex Gaussian elements with zero-mean and unit variance. The receive and transmit correlation matrices are arbitrarily spatially correlated.

The transmission takes place using OSTBCs that code $K$ symbols over $T$ symbols slots (i.e., the coding rate is $\frac{K}{T}$ ). Let $\mathbf{s}=$ $\left[s_{1}, \ldots, s_{K}\right]^{T} \in \mathbb{C}^{K}$ represent these $K$ data symbols, where each symbol $s_{i} \in \mathcal{A}$ has the average power $E\left\{\left|s_{i}\right|^{2}\right\}=\gamma$ and belongs to the constellation set $\mathcal{A}$ (different constellations will be considered). 
These symbols are coded in an OSTBC matrix $\mathbf{C}(\mathbf{s}) \in \mathbb{C}^{B \times T}$ that fulfills the orthogonality property $\mathbf{C}(\mathbf{s}) \mathbf{C}(\mathbf{s})^{H}=\|\mathbf{s}\|^{2} \mathbf{I}$, see [4] for details. The spatial coding dimension is $B$ and a linear precoder $\mathbf{W} \in \mathbb{C}^{n_{T} \times B}$ with the power constraint $\operatorname{tr}\left(\mathbf{W} \mathbf{W}^{H}\right)=1$ is used to project the code into advantageous spatial directions [5]. Observe that the precoder is normalized such that the average transmit power allocated per symbol is $E\left\{\|\mathbf{W C}(\mathbf{s})\|^{2}\right\} / k=\gamma$. Under these assumptions, the received signal $\mathbf{Y} \in \mathbb{C}^{n_{R} \times T}$ is

$$
\mathbf{Y}=\mathbf{H W C}(\mathbf{s})+\mathbf{N}
$$

where the power of the system has been normalized such that the additive white noise $\mathbf{N} \in \mathbb{C}^{n_{R} \times T}$ has i.i.d. complex Gaussian elements with zero-mean and unit variance. As shown in $[6,10]$, the use of OSTBCs makes it possible to decompose (2) into $K$ independent and virtual single-antenna systems as

$$
y_{k}^{\prime}=\|\mathbf{H W}\| s_{k}+n_{k}^{\prime}, \quad k=1, \ldots, K,
$$

where $n_{k}^{\prime}$ is complex Gaussian with zero-mean and unit variance.

\subsection{Expressions for the Symbol Error Rate}

Herein, the performance measure will be the SER; that is, the probability that the receiver makes an error in the detection of received symbols. The SER depends strongly on the signal-to-noise ratio (SNR) and the type of symbol constellation. Next, we will present SER expressions for some commonly considered constellations, but first we introduce a definition.

Definition 1. Let $\boldsymbol{\Phi} \triangleq \mathbf{R}_{R} \otimes\left(\mathbf{W} \mathbf{W}^{H} \mathbf{R}_{T}\right)^{T}$ and define the function

$$
F_{a, b}(g) \triangleq \frac{1}{\pi} \int_{a}^{b} \frac{d \theta}{\operatorname{det}\left(\mathbf{I}+\frac{\gamma g}{\sin ^{2}(\theta)} \mathbf{\Phi}\right)}, \quad g \geq 0, b \geq a
$$

We will consider the SER for three different types of constellations: PAM, PSK, and QAM. Let $g_{\mathrm{PAM}} \triangleq \frac{3}{M^{2}-1}, g_{\mathrm{PSK}} \triangleq$ $\sin ^{2}\left(\frac{\pi}{M}\right)$, and $g_{\mathrm{QAM}} \triangleq \frac{3}{2(M-1)}$. Then, the exact SER of the system in (3) was derived in $[11,6]$ and can be expressed as

$$
\begin{aligned}
\operatorname{SER}_{\mathrm{PAM}} & =\frac{2(M-1)}{M} F_{0, \frac{\pi}{2}}\left(g_{\mathrm{PAM}}\right) \\
\mathrm{SER}_{\mathrm{PSK}} & =F_{0, \frac{M-1}{M} \pi}\left(g_{\mathrm{PSK}}\right) \\
\mathrm{SER}_{\mathrm{QAM}} & =\frac{4(\sqrt{M}-1)}{M}\left(F_{0, \frac{\pi}{4}}\left(g_{\mathrm{QAM}}\right)+\sqrt{M} F_{\frac{\pi}{4}, \frac{\pi}{2}}\left(g_{\mathrm{QAM}}\right)\right)
\end{aligned}
$$

for $M$-PAM, $M$-PSK, and $M$-QAM constellations, respectively. Observe that the integral in Definition 1 is the main building stone in the SER expressions. The determinant in (4) can be expressed as

$$
\operatorname{det}\left(\mathbf{I}+\frac{\gamma g}{\sin ^{2}(\theta)} \boldsymbol{\Phi}\right)=\prod_{i=1}^{n_{T} n_{R}}\left(1+\frac{\gamma g}{\sin ^{2}(\theta)} \lambda_{i}(\boldsymbol{\Phi})\right)
$$

where $\lambda_{i}(\boldsymbol{\Phi})$ denotes the $i$ th largest eigenvalue of $\boldsymbol{\Phi}$. Hence, we conclude that the eigenvalues of $\boldsymbol{\Phi}$ (and not the eigenvectors) determines the SER. In the next sections we will analyze how the system performance depends on the spatial correlation and thus we focus on comparing systems with different eigenvalue distributions.

\subsection{Definitions of Majorization and Schur-Convexity}

The spatial correlation can be measured in the distribution of eigenvalues of the correlation matrices; low correlation is represented by eigenvalues that are almost identical, while high correlation means that a few eigenvalues are dominating. Herein, we assume that all eigenvalues are ordered in non-decreasing order and we will use the notion of majorization to compare systems [8]. If $\mathbf{x}=\left[x_{1}, \ldots, x_{M}\right]^{T}$ and $\mathbf{y}=\left[y_{1}, \ldots, y_{M}\right]^{T}$ are two non-negative vectors, then we say that $\mathbf{x}$ majorizes $\mathbf{y}$ if

$$
\sum_{k=1}^{m} x_{k} \geq \sum_{k=1}^{m} y_{k}, m=1, \ldots, M-1, \text { and } \sum_{k=1}^{M} x_{k}=\sum_{k=1}^{M} y_{k} .
$$

This property is denoted $\mathbf{x} \succeq \mathbf{y}$. If $\mathbf{x}$ and $\mathbf{y}$ contain eigenvalues, then $\mathbf{x} \succeq \mathbf{y}$ corresponds to that $\mathbf{x}$ is more spatially correlated than $\mathbf{y}$. Majorization only provides a partial order of vectors, but is still very powerful due to its connection to certain order-preserving functions:

A function $f(\cdot)$ is said to be Schur-convex if $f(\mathbf{x}) \geq f(\mathbf{y})$ for all $\mathbf{x}$ and $\mathbf{y}$, such that $\mathbf{x} \succeq \mathbf{y}$, and Schur-concave if $f(\mathbf{x}) \leq f(\mathbf{y})$.

\section{ANALYSIS OF THE SYMBOL ERROR RATE}

In this section, we will analyze the impact of spatial correlation on the system performance. The analysis is divided into two cases: without precoding and with SER minimizing precoding. Observe that all analytic results in this paper are derived for arbitrary functions of the type introduced in Definition 1 (and linear combinations of them). The three SER functions in (5) are just examples of functions of this type, so the results can potentially be used for other functions (either corresponding to SERs or something else).

In this section, we let the eigenvalues of the correlation matrices $\mathbf{R}_{T}$ and $\mathbf{R}_{R}$ be gathered in non-decreasing order in $\boldsymbol{\lambda}_{T} \in \mathbb{R}_{+}^{n_{T}}$ and $\boldsymbol{\lambda}_{R} \in \mathbb{R}_{+}^{n_{R}}$, respectively.

\subsection{Schur-Convexity Without Precoding}

First, we consider the case without linear precoding, represented by $B=n_{T}$ and $\mathbf{W}=\frac{1}{\sqrt{n_{T}}} \mathbf{I}$. Under these circumstances, the following theorem and its corollary show that the SER always increases with the spatial correlation (i.e., the performance is degraded).

Theorem 1. Consider the function $F_{a, b}(g)$ with $\mathbf{\Phi} \triangleq \mathbf{R}_{R} \otimes \frac{\mathbf{R}_{T}^{T}}{n_{T}}$. This function is Schur-convex with respect to $\boldsymbol{\lambda}_{T}$ when $\boldsymbol{\lambda}_{R}$ is fixed and Schur-convex with respect to $\lambda_{R}$ when $\lambda_{T}$ is fixed.

Proof. The proof follows by analyzing the integrand of (4) in a similar way as in the proof of Theorem 1 in [9].

Corollary 1. The SERs in (5) with M-PAM, M-PSK, and M-QAM are all Schur-convex with respect to the receive correlation for fixed transmit correlation, and vice versa.

\subsection{Schur-Convexity With Optimal Precoding}

Next, we consider the case when the precoder $\mathbf{W}$ is chosen to minimize the SER. For this purpose, we define the following function.

Definition 2. Let $\mathbf{\Phi} \triangleq \mathbf{R}_{R} \otimes\left(\mathbf{W} \mathbf{W}^{H} \mathbf{R}_{T}\right)^{T}$ and define the function

$$
G_{a, b, c, d}(g) \triangleq \min _{\mathbf{W}, \operatorname{tr}\left(\mathbf{W} \mathbf{W}^{H}\right)=1} C_{1} F_{a, b}(g)+C_{2} F_{c, d}(g),
$$

where $C_{1}$ and $C_{2}$ are non-negative constants, $b \geq a$, and $d \geq c$. 
Observe that all the SER expressions in (5) can be expressed as $G_{a, b, c, d}(g)$ when SER minimizing precoding has been applied. Let the eigenvalue decomposition of the transmit correlation matrix be $\mathbf{R}_{T}=\mathbf{U}_{T} \boldsymbol{\Lambda}_{T} \mathbf{U}_{T}^{H}$, where the diagonal matrix $\boldsymbol{\Lambda}_{T}$ contains the eigenvalues in non-decreasing order and the unitary matrix $\mathbf{U}_{T}$ contains the corresponding eigenvectors. It is known that the precoder that gives $G_{a, b, c, d}(g)$ (i.e., the SER minimizing precoder) can be expressed as $\mathbf{W}=\mathbf{U}_{T} \boldsymbol{\Delta}$, where $\boldsymbol{\Delta} \in \mathbb{C}^{n_{T} \times B}$ is a rectangular diagonal matrix [6]. Hence, we have that $\mathbf{W} \mathbf{W}^{H}=\mathbf{U}_{T} \boldsymbol{\Lambda}_{W} \mathbf{U}_{T}^{H}$, where $\boldsymbol{\Lambda}_{W} \triangleq \boldsymbol{\Delta} \boldsymbol{\Delta}^{H}=\operatorname{diag}\left(p_{1}, \ldots, p_{B}, 0, \ldots, 0\right)$ represents the power assigned to different transmit eigenmodes. The following lemma gives the asymptotically optimal precoders at low and high SNRs (the latter was proved in [6] in the case of correlation with full rank).

Lemma 1. Consider the function $G_{a, b, c, d}(g)$ and let the optimal precoder be denoted $\mathbf{W}$. Let the SNR be represented by $\gamma$ and let $\mathbf{R}_{T}=\mathbf{U}_{T} \boldsymbol{\Lambda}_{T} \mathbf{U}_{T}^{H}$ be the eigenvalue decomposition of $\mathbf{R}_{T}$, where the diagonal matrix $\boldsymbol{\Lambda}_{T}$ contains the eigenvalues in nondecreasing order and the unitary matrix $\mathbf{U}_{T}$ contains the corresponding eigenvectors. Then, $\mathbf{W}$ is given by $\mathbf{W}=\mathbf{U}_{T} \boldsymbol{\Delta} \widetilde{\mathbf{U}}$, where $\widetilde{\mathbf{U}} \in \mathbb{C}^{B \times B}$ is an arbitrary unitary matrix and $\boldsymbol{\Lambda}_{W} \triangleq \Delta \boldsymbol{\Delta}^{H}$ is diagonal and has $\operatorname{rank}\left(\boldsymbol{\Lambda}_{W}\right) \leq B$. The optimal power allocation at low $\operatorname{SNR}$ is $\boldsymbol{\Lambda}_{W}=\operatorname{diag}(1,0, \ldots, 0)$ (i.e., selective allocation to the strongest eigenmode), while the allocation is $\boldsymbol{\Lambda}_{W}=$ $\operatorname{diag}\left(\frac{1}{\widetilde{B}}, \ldots, \frac{1}{\widetilde{B}}, 0, \ldots, 0\right)$ at high $S N R$ (i.e., equal allocation to the $\tilde{B}$ dominating eigenmodes) where $\widetilde{B}=\min \left(B, \operatorname{rank}\left(\mathbf{R}_{T}\right)\right)$.

For an SER minimizing precoder, the following theorem and its corollary show how the SER behaves with respect to the spatial correlation. The asymptotic results of Lemma 1 play an important role since the Schur-convexity properties change with the SNR.

Theorem 2. The function $G_{a, b, c, d}(g)$ is Schur-convex with respect to $\boldsymbol{\lambda}_{R}$ (when $\boldsymbol{\lambda}_{T}$ is fixed) independently of the SNR. The function is Schur-convex with respect to $\lambda_{T}$ (when $\lambda_{R}$ is fixed) at high SNR, while it is Schur-concave at low SNR.

Proof. The proof follows from Schur's condition [8] and Lemma 1 by differentiation of $G_{a, b, c, d}(g)$ and some identification.

Corollary 2. Consider the SERs in (5) with M-PAM, M-PSK, and $M-Q A M$ when an SER minimizing linear precoder is used. These function are Schur-convex with respect to the receive correlation (for fixed transmit correlation). They are also Schur-convex with respect to the transmit correlation (for fixed received correlation) at high SNR, but Schur-concave at low SNR.

From Corollary 2, we draw the conclusion that even with an optimal precoder, correlation at the receiver side will always degrade the performance. The combination of SER minimizing precoding and spatial correlation at the transmitter side will however improve the performance at low SNR, while correlation still might be bad at high SNR. Thus, it is of practical importance to quantify what low SNR actually means in this context. An indication is given by the following lemma that treats an upper bound on $F_{a, b}(g)$.

Lemma 2. Consider the following upper bound on $F_{a, b}(g)$ :

$$
F_{a, b}(g) \leq \frac{1}{\pi} \frac{b-a}{\operatorname{det}(\mathbf{I}+\gamma g \boldsymbol{\Phi})}
$$

The expression on the right hand side of (8) is Schur-concave with respect to the transmit correlation (for fixed receive correlation) for all SNR $\gamma$ such that $\gamma \leq \frac{1}{g \operatorname{tr}\left(\mathbf{R}_{T}\right) \operatorname{tr}\left(\mathbf{R}_{R}\right)}$.

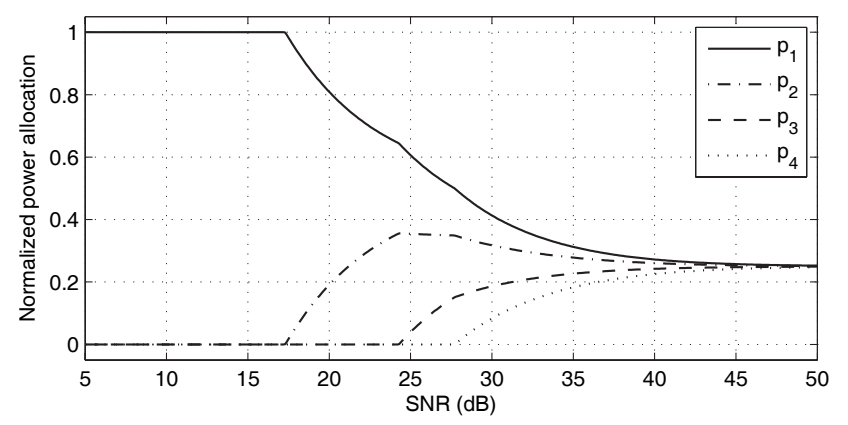

Fig. 1. The SER minimizing (normalized) power allocation $\boldsymbol{\Lambda}_{W}=$ $\operatorname{diag}\left(p_{1}, p_{2}, p_{3}, p_{4}\right)$ as a function of the SNR. The transmitter and the receiver are equipped with four antennas, with a fixed correlation of 0.5 between adjacent antennas in each array [12].

Proof. The expression in (8) is the Chernoff bound on $F_{a, b}(g)$. The Schur-concavity follows from Schur's condition [8], Lemma 1, and a Maclaurin expansion that is valid for certain $\gamma$.

Next, the results of this section will be illustrated numerically and it will become apparent that the SER is (at least approximately) Schur-concave with respect to the transmit correlation for a much larger SNR region than the one stated in Lemma 2.

\section{NUMERICAL EXAMPLES}

In this section, the precoder properties of Lemma 1 and the Schurconvexity results of Corollary 2 will be illustrated numerically. We consider a system where the transmitter and the receiver are equipped with four antennas each. The antenna correlation follows the exponential model [12], which in principle models a uniform linear array (ULA) with the correlation between adjacent antenna elements as a parameter. An OSTBC with rate $3 / 4$ and a spatial coding dimension of 4 is used, for example the one proposed in [4], and the symbol constellation is 16-QAM. For normalization purposes, we let $\operatorname{tr}\left(\mathbf{R}_{T}\right)=\operatorname{tr}\left(\mathbf{R}_{R}\right)=1$. Thus, the transmit power (per data symbol) $\gamma$ also equals the received SNR (with equal power allocation) and the transmitted SNR.

Let the power allocation of the SER minimizing precoder be denoted $\boldsymbol{\Lambda}_{W}=\operatorname{diag}\left(p_{1}, p_{2}, p_{3}, p_{4}\right)$, where $p_{k}$ is applied to the $k$ th strongest transmit eigenmode. In Fig. 1, the SER minimizing power allocation is given as a function of the SNR (in terms of $\gamma$ ). The antenna correlation is fixed at 0.5 at both sides, which corresponds to the eigenvalue distribution $[0.5214,0.2500,0.1349,0.0938]^{T}$. In line with Lemma 1, we observe that all power is allocated to the dominating eigenmode at low SNRs and that the system approaches equal power allocation at high SNRs. In between these extremes, the optimal power allocation has the typical waterfilling behavior.

Next, we keep the transmit correlation fixed at 0.5 , while the correlation between adjacent receive antennas is changed between 0 and 1 (i.e., completely uncorrelated and correlated, respectively). The SER with SER minimizing precoding is shown in Fig. 2 at different SNRs. It is seen that the SER is Schur-convex with respect to the receive correlation eigenvalues at all SNRs, which confirms the results of Corollary 2.

Finally, we keep the receive correlation fixed at 0.5 , while the transmit correlation is changed from the completely uncorrelated to the completely correlated case. The SER with SER minimizing precoding is shown in Fig. 3 at different SNRs. At 0, 15, and $25 \mathrm{~dB}$, the 
SER is Schur-concave (i.e., decreases with the spatial correlation). At very high SNR (e.g., $35 \mathrm{~dB}$ ), the SER is Schur-convex, while there is a transisition interval when the SER first increases with the correlation and then decreases again.

From Lemma 2, we expect the low $S N R$-region (where $p_{1}=1$ and the SER is Schur-concave with respect to the transmit correlation) to include $\gamma \leq 10 \mathrm{~dB}$. In comparsion with the numerical examples, this condition seems rather strict. In Fig. 1, selective power allocation (i.e, beamforming) is optimal for $\gamma \leq 17 \mathrm{~dB}$, and it is seen in Fig. 3 that the SER is Schur-concave for SNRs up to at least $25 \mathrm{~dB}$. An important observation is that when the SER transistions from a Schur-concave function to a Schur-convex function, it has already reached such low values (below $10^{-6}$ ) that the dependence on the spatial correlation is negligible. Thus, we conclude that the SER is Schur-concave at low to medium SNRs and approximately Schur-concave at high SNRs.

\section{CONCLUSIONS}

In this paper, the performance of spatially correlated Kroneckerstructured MIMO systems with orthogonal space-time block coding has been analyzed in terms of the symbol error rate. The results cover the exact SER for $M$-PAM, $M$-PSK, and $M$-QAM, but also a wider range of functions that may correspond the SERs for other constellations. Using majorization and the notion of Schurconvexity, it has been shown analytically that the SER is Schurconvex with respect to the spatial correlation at the receiver side (i.e., the error increases with the correlation). This holds both in the case without precoding and with an SER minimizing linear precoder. The SER is also Schur-convex with respect to the transmit correlation without precoding, while transmit correlation actually improves the performance when an SER minimizing linear precoder is used. Analytically, the SER is Schur-concave with respect to the transmit correlation at low SNRs (i.e., the error decreases with increasing transmit correlation) while it still becomes Schur-convex at high SNRs. An approximate lower bound on the SNR region of Schur-concavity has been derived, but as illustrated numerically, the SER is actually approximately Schur-concave at all SNRs.

The SER minimizing precoder scales the eigenvalues of the transmit correlation matrix, and the statistical waterfilling behavior of these scaling factors has been illustrated numerically. Finally, it has been shown that selective and equal power allocation is asymptotically optimal at low and high SNRs, respectively.

\section{REFERENCES}

[1] E. Telatar, "Capacity of multi-antenna Gaussian channels," European Trans. Telecom., vol. 10, pp. 585-595, 1999.

[2] E. Björnson and B. Ottersten, "Training-based Bayesian MIMO channel and channel norm estimation," in Proc. IEEE ICASSP'09, 2009.

[3] M. Vu and A. Paulraj, "MIMO wireless linear precoding," IEEE Signal Processing Magazine, vol. 24, no. 5, pp. 86-105, 2007.

[4] V. Tarokh, H. Jafarkhani, and A.R. Calderbank, "Space-time block codes from orthogonal designs," IEEE Trans. Inf. Theory, vol. 45, pp. 1456-1467, 1999.

[5] G. Jöngren, M. Skoglund, and B. Ottersten, "Combining beamforming and orthogonal space-time block coding," IEEE Trans. Inf. Theory, vol. 48, pp. 611-627, 2002.

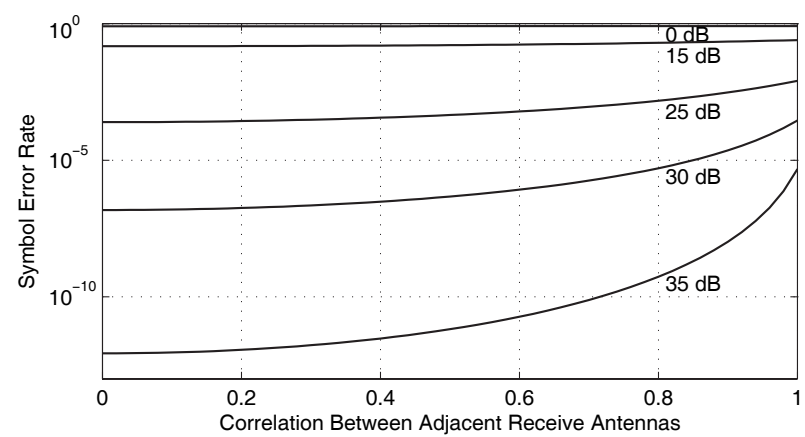

Fig. 2. The SER as a function of the correlation between adjacent receive antennas in a four-antenna array [12]. The transmitter has four antennas with the corresponding fixed correlation of 0.5 . The Schur-convexity properties of the SER are shown at different SNRs.

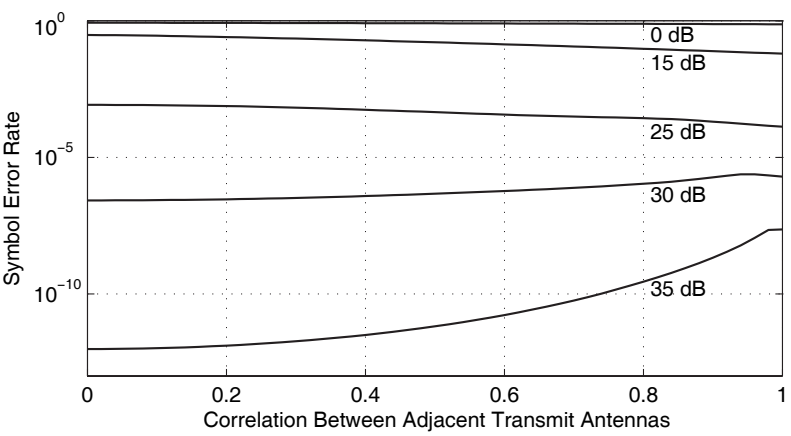

Fig. 3. The SER as a function of the correlation between adjacent transmit antennas in a four-antenna array [12]. The receiver has four antennas with the corresponding fixed correlation of 0.5 . The Schurconvexity properties of the SER are shown at different SNRs.

[6] A. Hjørungnes and D. Gesbert, "Precoding of orthogonal space-time block codes in arbitrarily correlated MIMO channels: Iterative and closed-form solutions," IEEE Trans. Wireless Commun., vol. 6, pp. 1072-1082, 2007.

[7] K.T. Phan, S.A. Vorobyov, and C. Tellambura, "Precoder design for space-time coded MIMO systems with correlated Rayleigh fading channels," in Proceedings of Canadian Conference on Electrical and Computer Engineering, 2007.

[8] E. Jorswieck and H. Boche, "Majorization and matrixmonotone functions in wireless communications," Foundations and Trends in Communication and Information Theory, vol. 3, pp. 553-701, 2007.

[9] E. Björnson, P. Devarakota, S. Medawar, and E. Jorswieck, "Schur-convexity of the symbol error rate in correlated MIMO systems with precoding and space-time coding," in Proc. Nordic Radio Science and Commun. (RVK'08), 2008.

[10] S. Sandhu and A. Paulraj, "Space-time block codes: A capacity perspective,” IEEE Commun. Lett., vol. 4, pp. 384-386, 2000.

[11] M.K. Simon and M. Alouini, "A unified approach to the performance analysis of digital communication over generalized fading channels," Proc. IEEE, vol. 86, pp. 1860-1877, 1998.

[12] S.L. Loyka, "Channel capacity of MIMO architecture using the exponential correlation matrix," IEEE Commun. Lett., vol. 5, pp. 369-371, 2001. 